\title{
Comprehensive analysis of circulating microRNAs as predictive biomarkers for sorafenib therapy outcome in hepatocellular carcinoma
}

\author{
TOMOKI KOHNO ${ }^{1}$, ASAHIRO MORISHITA ${ }^{1}$, HISAKAZU IWAMA ${ }^{2}$, KOJI FUJTAA $^{1}$, JOJI TANI $^{1}$, KEI TAKUMA ${ }^{1}$, \\ MAI NAKAHARA ${ }^{1}$, KYOKO OURA ${ }^{1}$, TOMOKO TADOKORO ${ }^{1}$, TAKAKO NOMURA ${ }^{1}$, HIROHITO YONEYAMA ${ }^{1}$, \\ KIYOHITO KATO $^{1}$, KEIICHI OKANO ${ }^{3}$, YASUYUKI SUZUKI ${ }^{3}$, AKIRA NISHIYAMA ${ }^{4}$, \\ TAKASHI HIMOTO ${ }^{5}$ and TSUTOMU MASAKI ${ }^{1}$ \\ ${ }^{1}$ Department of Gastroenterology and Neurology; ${ }^{2}$ Life Science Research Center; Departments of \\ ${ }^{3}$ Gastroenterological Surgery and ${ }^{4}$ Pharmacology, Kagawa University Faculty of Medicine, Kita-gun, Kagawa 761-0793; \\ ${ }^{5}$ Department of Medical Technology, Kagawa Prefectural University of Health Sciences, Takamatsu, Kagawa 761-0123, Japan
}

Received July 25, 2019; Accepted December 17, 2019

DOI: 10.3892/ol.2020.11696

\begin{abstract}
Hepatocellular carcinoma (HCC) is the third leading cause of cancer-related death worldwide. Clinical management has improved the prognosis of early $\mathrm{HCC}$, but that of advanced HCC remains poor. Sorafenib, an oral multikinase inhibitor, provided a treatment option for advanced-stage HCC, and prolonged the survival and inhibited tumor progression as first-line therapy in patients with advanced HCC. In this study, we investigated if specific microRNAs could act as predictive biomarkers of sorafenib effectiveness and indicate the best time to switch to second-line therapies. Sorafenib inhibited the proliferation of the Li-7, Hep3B, HepG2 and Huh7 liver cancer cell lines (effective group), but not that of the HLE, HLF and ALEX cancer cell lines (non-effective group). A microRNA (miRNA/miR) analysis was performed comparing sorafenib-effective and non-effective cells lines as well as serum samples from patients with HCC from sorafenib-effective (complete response/partial response) and -non-effective (progressive disease) groups before sorafenib administration and detected three differentially-expressed miRNAs that were common among the in vivo and in vitro samples. The increase rate (effective/non-effective) of hsa-miR-30d in the medium was higher than that in the cancer cells. hsa-miR-30d was highly expressed in the serum and exosomes of patients with
\end{abstract}

Correspondence to: Dr Asahiro Morishita, Department of Gastroenterology and Neurology, Kagawa University Faculty of Medicine, 1750-1 Ikenobe, Miki-cho, Kita-gun, Kagawa 761-0793, Japan

E-mail: asahiro@med.kagawa-u.ac.jp

Abbreviations: miRNA, microRNA; HCC, hepatocellular carcinoma; $\mathrm{CR}$, complete response; $\mathrm{PR}$, partial response; $\mathrm{SD}$, stable disease

Key words: miRNA, sorafenib, HCC, hsa-miR-30d, predictive biomarker, regorafenib
HCC in the effective group when compared to those of the non-effective group. Additionally, the hsa-miR-30d expression in the medium of cancer cell lines was highly upregulated in the effective group compared with the non-effective group. These results suggested that hsa-miR-30d might be secreted by the cancer cells to the serum through the exosomes. We identified a specific circulating miRNA that is related to refractory HCC under sorafenib therapy. Therefore, hsa-miR-30d might serve as a predictive biomarker for the efficacy of sorafenib therapy in $\mathrm{HCC}$.

\section{Introduction}

Hepatocellular carcinoma (HCC) is the third leading cause of cancer-related death (1), in spite of recent improvements in risk factor regulation and surveillance (2). Surgical resection is the first choice to treat early-stage HCC. However, the 5-year recurrence rate after hepatectomy reaches approximately $70 \%$ (3). Therefore, most cases of recurrent HCC develop to advanced stages (4). The prognosis of early-stage HCC has improved thanks to clinical management, but that of advanced-stage HCC remains extremely poor (5). The targeted agent sorafenib, an oral multikinase inhibitor, was introduced as first-line systemic therapy for HCC in 2007 (6). Sorafenib therapy provided an additional treatment option for patients with advanced HCC who had local vascular invasion or distant metastasis (6). Sorafenib prolonged the median survival and time to radiologic progression in patients with advanced HCC for 3 months over that of placebo (7). Recently, regorafenib, a multiple receptor tyrosine kinase inhibitor, was approved for the treatment of advanced HCC that has progressed after sorafenib therapy (8). Therefore, predictive biomarkers of the outcomes of sorafenib therapy that indicate the best time to switch to second-line therapy should be assessed before starting treatment. However, no biological serum marker has been discovered for the prediction of refractory HCC under sorafenib therapy.

MicroRNAs (miRNAs) are 18-22 nucleotide-long endogenous noncoding RNAs $(9,10)$ that regulate over 
200 genes (11). Previous reports have shown altered expression of several miRNAs in HCC tissues when compared to normal tissues (12-15). Recently, miRNAs have been evaluated as tissue biomarkers in the context of sorafenib response in $\operatorname{HCC}(16,17)$.

In this study, we evaluated whether specific circulating miRNAs were involved with refractory HCC under sorafenib therapy to identify miRNAs that may serve as new predictive biomarkers for the efficacy of sorafenib therapy in this disease.

\section{Materials and methods}

Cell culture. The human liver cancer cell lines Li-7, Hep3B, HepG2, HLE, HLF, Alex and Huh7 were obtained from the Japanese Cancer Research Resources Bank and transported to our laboratory. The cell lines were authenticated by the cell bank using short tandem repeat PCR. Mycoplasma testing has been done for cell lines used in our experiments. Cells were grown in minimum essential medium (MEM; Gibco-Invitrogen) supplemented with 10\% fetal bovine serum (FBS, 533-69545; Wako) and penicillin-streptomycin (100 mg/l; Invitrogen) at $37^{\circ} \mathrm{C}$ in a humidified atmosphere containing $5 \% \mathrm{CO}_{2}$.

Cell proliferation assay. The cell proliferation assays were conducted using CCK- 8 according to the manufacturer's instructions. Each cell line $\left(0.5 \times 10^{4}\right)$ was seeded on 96 -well plates and cultured in $100 \mu \mathrm{l}$ MEM supplemented with $10 \%$ FBS. After $24 \mathrm{~h}$, seeded cells were treated with $0,1,3,5$, or $10 \mu \mathrm{M}$ sorafenib that was added to the culture medium. At the indicated time points, the medium was exchanged for $100 \mu \mathrm{l}$ of MEM with CCK-8 reagent (10 $\mu \mathrm{l} \mathrm{CCK-8} \mathrm{and} 90 \mu \mathrm{l}$ MEM). The absorbance of each well was measured at a wavelength of $450 \mathrm{~nm}$ using an auto-microplate reader.

Patients and serum samples. We obtained serum samples from 11 patients with advanced HCC who underwent sorafenib therapy at the Kagawa University hospital from 2012 to 2015 . All samples were drawn before sorafenib administration (Table I). This study was conducted in accordance with the ethical principles of the Declaration of Helsinki and approved by The Institutional Review Board (IRB) of Kagawa University, Faculty of Medicine (Heisei-22-063). Informed consent to use the clinical data and samples for the present study was obtained from the patients or their relatives. For patients who died and had no relatives listed in their clinical records, we provided opt-out methods for the relatives of the dead participants by publishing a summary of this study on the university website. Ethics approval was obtained from The Ethics Committee of Kagawa University Faculty of Medicine.

miRNA microarray for liver cancer cell lines and serum samples from advanced HCC patients. Total miRNA was extracted from liver cancer cell lines using the Qiagen miRNeasy kit (Qiagen K.K.) according to the instructions provided by the manufacturer. Each serum sample was processed for total RNA extraction with the miRNeasy Mini Kit (Qiagen) according to the manufacturer's instructions. The RNA sample from both sets typically showed $A_{260 / 280}$ ratios between 1.9 and 2.1 measured with an Agilent 2100 Bioanalyzer (Agilent Technologies).
After RNA quantification with an RNA 6000 Nano kit (Agilent Technologies), the samples were labeled using a miRCURY Hy3/Hy5 Power labeling kit and hybridized on a human miRNA Oligo chip10, version 14.0 (Toray Industries). Scanning was conducted with the 3D-Gene Scanner 3000 (Toray Industries). The 3D-Gene extraction version 1.2 software (Toray Industries) was used to read the raw intensity of the image. The raw data were analyzed with GeneSpringGX v 10.0 (Agilent Technologies) to determine the change in miRNA expression. The samples were frozen at $-80^{\circ} \mathrm{C}$ within $4 \mathrm{~h}$ of collection and thawed just before analysis.

Reverse transcription (RT)-PCR for quantifying miRNA in liver cancer cells and circulating miRNA in serum samples. Total miRNA was extracted from liver cancer cells using the QIAGEN miRNeasy kit (Qiagen K.K.) for miRNA quantification according to the instructions provided by the manufacturer. Total RNA was reverse-transcribed using TaqMan MicroRNA Reverse Transcription kit (Life Technologies Japan). RNU6B was used as an internal control for relative quantification of hsa-miR-30d.

Circulating miRNA was extracted from $200 \mu 1$ of serum samples using the Qiagen miRNeasy serum-plasma kit (Qiagen K.K.) according to the instructions provided by the manufacturer. RNA was reverse-transcribed using the TaqMan MicroRNA Reverse Transcription kit (Life Technologies Japan) following the manufacturer's instructions. Caenorhabditis elegans miR-39 (cel-miR-39) was spiked in each sample as a control for the extraction and amplification steps. Serum miRNA was amplified using primers and probes provided by Applied Biosystems by the TaqMan MicroRNA assay, according to the instructions provided by the manufacturer. The relative expression of serum miRNA was calculated using the comparative cycle quantification $(\mathrm{Cq})$ method $\left(2^{-\Delta \Delta \mathrm{Cq}}\right)(18)$ with spiked cel-miR-39 as a normalized internal control.

Statistical analysis. Replicate data were consolidated for each sample group as follows; differences between the effective and non-effective groups i) in vivo and ii) in vitro. The data were organized using the hierarchical clustering and ANOVA functions in the GeneSpring software. Hierarchical clustering was performed using the clustering function (condition tree) and Euclidean correlation as a distance metric. Two-way ANOVA with Bonferroni's correction and asymptotic P-value $(<0.05)$ computation without any error correction on the samples were conducted to search for the miRNAs with the most prominent variation across the different groups. All analyzed data were scaled by global normalization. The statistical significance of differentially-expressed miRNAs was analyzed by Mann-Whitney U test. All analyses were conducted using computer-assisted JMP8.0 (SAS Institute). Paired analysis between the groups was conducted using the Student's t-test and Fisher's exact test. $\mathrm{P}<0.01$ (Cluster analysis in vitro) and $\mathrm{P}<0.05$ (Cluster analysis in vivo) were considered to indicate a significant difference between groups.

\section{Results}

Sorafenib inhibits cell proliferation of Li-7, Нер3B, HepG2, and Huh7, but not of HLE, HLF, and ALEX cells. We examined 
Table I. Baseline characteristics of the patients.

\begin{tabular}{lccc}
\hline Characteristics & Effective (n=6) & Non-effective (n=5) & P-values \\
\hline Age (median) & $69(53-79)$ & $68(55-76)$ & $\mathrm{NS}$ \\
Sex, male/female & $5 / 1$ & $4 / 1$ & $\mathrm{NS}$ \\
Etiology, B/C/NBNC & $1 / 3 / 2$ & $2 / 3 / 1$ & $\mathrm{NS}$ \\
Stage, III/IVa/IVb & $2 / 3 / 1$ & $5 / 1 / 0$ & $\mathrm{NS}$ \\
Child-Pugh, A/B/C & $5 / 1 / 0$ & $4 / 1$ & $\mathrm{NS}$ \\
Vessel invasion, none/portal vein & $4 / 2$ & $4 / 1$ & $\mathrm{NS}$ \\
Reason of sorafenib administration, TACE & $4 / 2$ & & $\mathrm{NS}$ \\
refractory/vascular invasion & & $1 / 3 / 1$ & $\mathrm{NS}$ \\
Initial dose, 200/400/800 & $1 / 4 / 1$ & $2 / 3$ & $\mathrm{NS}$ \\
Primary/recurrent & $3 / 3$ & & \\
\hline
\end{tabular}

TACE, transarterial chemoembolization; NS, not significant.

A Li-7
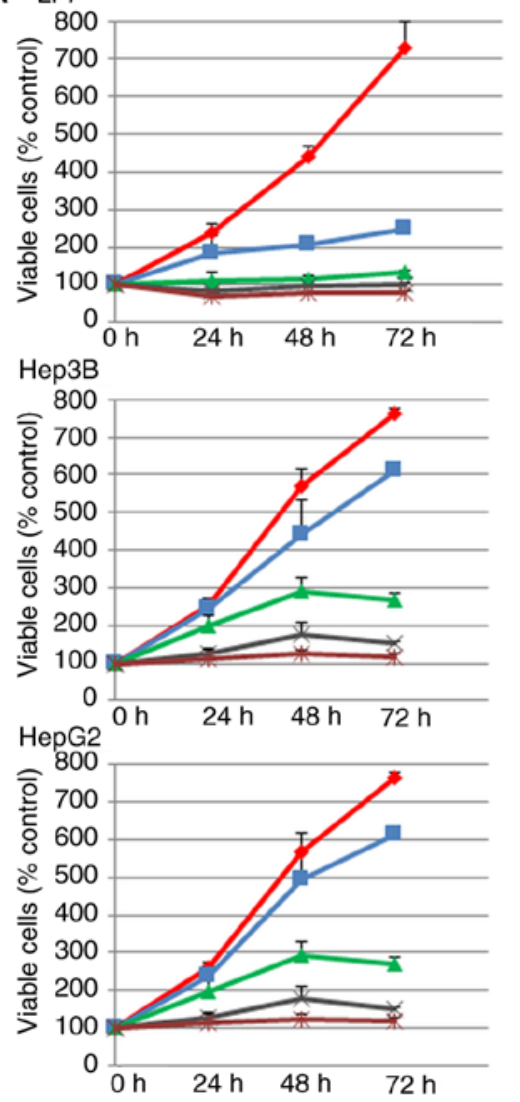

B HLE
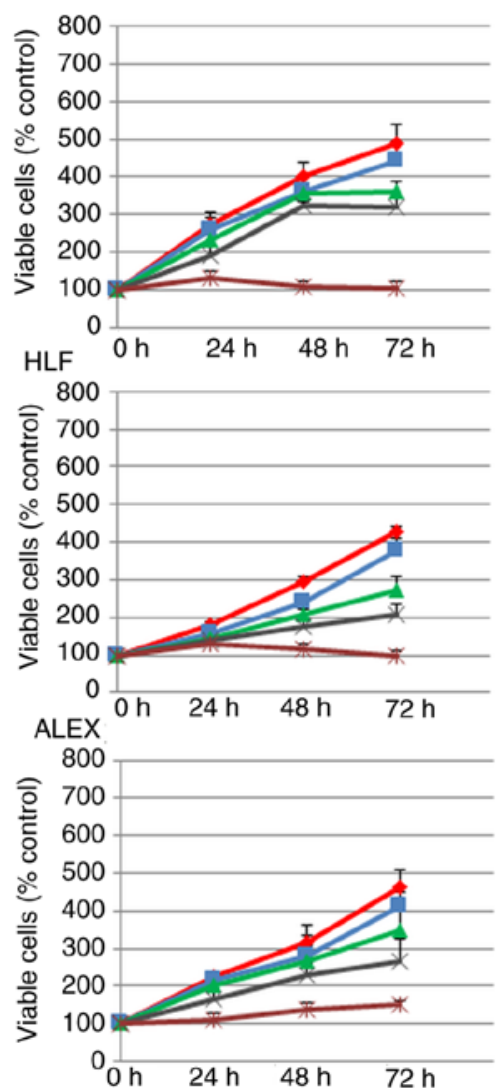

C Huh7
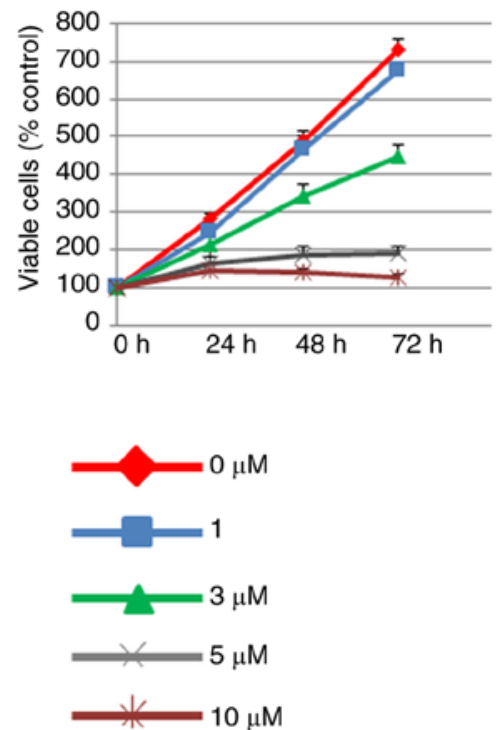

Figure 1. MTT assay showing the effect of sorafenib administration in vitro. (A) Effective group represented by Li-7, Hep3B and HepG2 cell lines. (B) Non-effective group represented by HLE, HLF and ALEX cell lines. (C) Huh7 was partially inhibited by sorafenib and therefore, was not sorted in any group.

the in vitro effects of sorafenib on seven human liver cancer cell lines, Li-7, Hep3B, HepG2, HLE, HLF, Alex and Huh7 by following the course of proliferation of each of them for three days after sorafenib addition and evaluating the direct relationship between the decrease of cell viability and the inhibition of cell proliferation. Cells were cultured and with 0,1 , 3 or $10 \mu \mathrm{M}$ sorafenib was added to the medium after $24 \mathrm{~h}$. As shown in Fig. 1, sorafenib strongly inhibited the proliferation of Li-7, Hep3B, and HepG2 cells in a dose- and time-dependent manner (sorafenib-effective group), but not that of HLE, HLF, and ALEX cells (sorafenib non-effective group). Huh7 cell growth was partially inhibited by sorafenib (Fig. 1), so it was not included in either group. These results demonstrated that sorafenib inhibits the proliferation of certain human liver cancer cell lines.

Comparison of miRNA expression in cancer cell lines between sorafenib-effective and non-effective groups. A miRNA array 


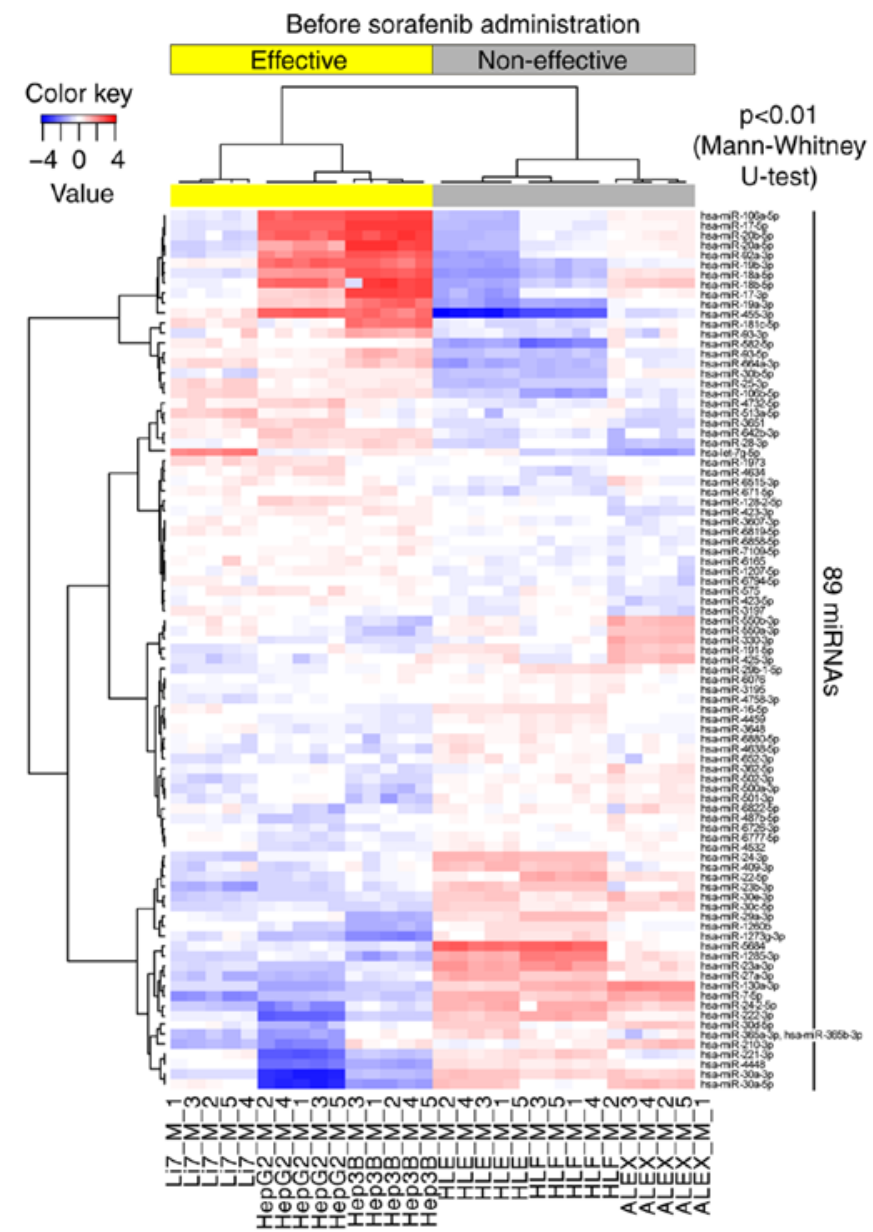

Figure 2. Cluster analysis of miRNA expression between the effective and non-effective group before sorafenib administration in vitro. The sorafenib-effective group formed a cluster separated from the non-effective group, 89 miRNAs were significantly up- or down-regulated between the groups. miRNA, microRNA.

was performed in all liver cancer cell lines before sorafenib administration, and the results of the sorafenib-effective group were compared to those of the non-effective group. We examined the expression patterns of 2555 miRNAs extracted from the cell lines. The unsupervised hierarchical clustering analysis, using Pearson's correlation, showed that the sorafenib-effective group formed a cluster separated from that of the non-effective group (Fig. 2). Furthermore, 89 miRNAs were significantly differentially expressed between the groups (Fig. 2).

Patient characteristics in the sorafenib-effective and non-effective groups. We included the serum samples of 11 patients ( 9 males and 2 females with a median age of 69 years, ranging between 53 and 79 years) in the miRNA analysis. All patients received sorafenib therapy. The parameter association between the effective and non-effective groups is summarized in Table I. The analysis of age, sex, etiology, tumor stage, Child-Pugh classification, vessel invasion, reason of sorafenib administration, initial sorafenib dose, and primary or recurrent cancer were conducted using various categories of the Student's t-test and Fisher's exact test. No statistically significant differences were observed between the effective and non-effective groups regarding patient background.
Comparison of miRNA expression in serum samples of HCC patients before sorafenib administration between the sorafenib-effective and non-effective groups. We also performed miRNA array using serum samples from patients with HCC and compared the results of the sorafenib-effective and non-effective groups (Fig. 3). The unsupervised hierarchical clustering analysis, using Pearson's correlation, showed that the effective group formed a cluster separated from that of the non-effective group. Additionally, 10 miRNAs had significantly different expression patterns in the sorafenib-effective group when compared to the non-effective group.

Statistical analysis of miRNA expression between cancer cell lines and serum samples of HCC patients. We detected 3 miRNAs that were significantly changed between the sorafenib-effective and non-effective groups jointly in the in vitro and in vivo experiments (Table II). The hsa-miR-296-5p was up-regulated in the effective groups of both the liver cancer cell lines and the serum samples of HCC patients. The hsa-miR-6729-5p was down-regulated in the effective groups of both sets of samples. In contrast, hsa-miR-30d was down-regulated in the effective group of the liver cancer cell lines but up-regulated in the serum samples (Table II). Among 3 miRNAs, hsa-miR-30d might not be leaked, but actively secreted from the cancer cell.

To determine if hsa-miR-30d was secreted from the liver cancer cell to the extracellular fluid, we compared its expression levels in the serum of patients with HCC before sorafenib administration in the sorafenib-effective and non-effective groups using real-time quantitative (RTq-PCR). hsa-miR-30d expression was up-regulated in the serum and exosomes of $\mathrm{HCC}$ patients of the effective group when compared to that of the non-effective group ( $\mathrm{P}=0.0065$ and ${ }^{\#} \mathrm{P}=0.0464$, respectively, Fig. 4A and B). We also quantified hsa-miR-30d expression in the medium of liver cancer cells by RTq-PCR and found that HCC cells of the effective group up-regulated hsa-miR-30d when compared to the non-effective group $\left({ }^{\$} \mathrm{P}=0.0021\right.$, Fig. 4C).

\section{Discussion}

HCC is one of the most common forms of cancer worldwide (19), and the prognosis of patients in advanced stages remains very poor (5). Sorafenib, an oral multikinase inhibitor that suppresses angiogenesis in advanced-stage HCC, was the first drug approved to treat unresectable HCC (20). A recent clinical trial demonstrated that sorafenib is an effective first-line systemic therapy for patients with advanced HCC, but that only a subset of patients responds to the treatment (20). Regorafenib was recently approved as second-line systemic therapy for the treatment of advanced HCC that has progressed after sorafenib therapy (8). Therefore, biomarkers that predict the efficacy of sorafenib therapy and allow to switch smoothly from sorafenib to regorafenib are needed.

In the present study, we determined that circulating hsa-miR-30d is a potential predictive biomarker for sorafenib response when measured in the serum samples of HCC patients (Figs. 3 and 4; Table II). Recently, miRNAs such as miR-181a-5p and miR-339-5p, have been shown to predict early response to sorafenib treatment (21). Pretreatment-circulating miRNAs such as miR-21, miR-18a, miR-221, miR-139-5p, 
Table II. Statistical analysis of miR expressions between cancer cell lines and serum samples of patients with hepatocellular carcinoma.

\begin{tabular}{|c|c|c|c|c|}
\hline \multirow[b]{3}{*}{ Targeted miR } & \multicolumn{2}{|r|}{ Cell } & \multicolumn{2}{|c|}{ Serum } \\
\hline & & Fold Change & & Fold Change \\
\hline & P-values & Effective/Non-effective & P-values & Effective/Non-effective \\
\hline hsa-miR-296-5p & 0.049 & 1.175 & 0.044 & 1.402 \\
\hline hsa-miR-30d-5p & 0.0004 & 0.513 & 0.017 & 2.540 \\
\hline hsa-miR-6729-5p & 0.029 & 0.888 & 0.016 & 0.860 \\
\hline
\end{tabular}

miR, microRNA.

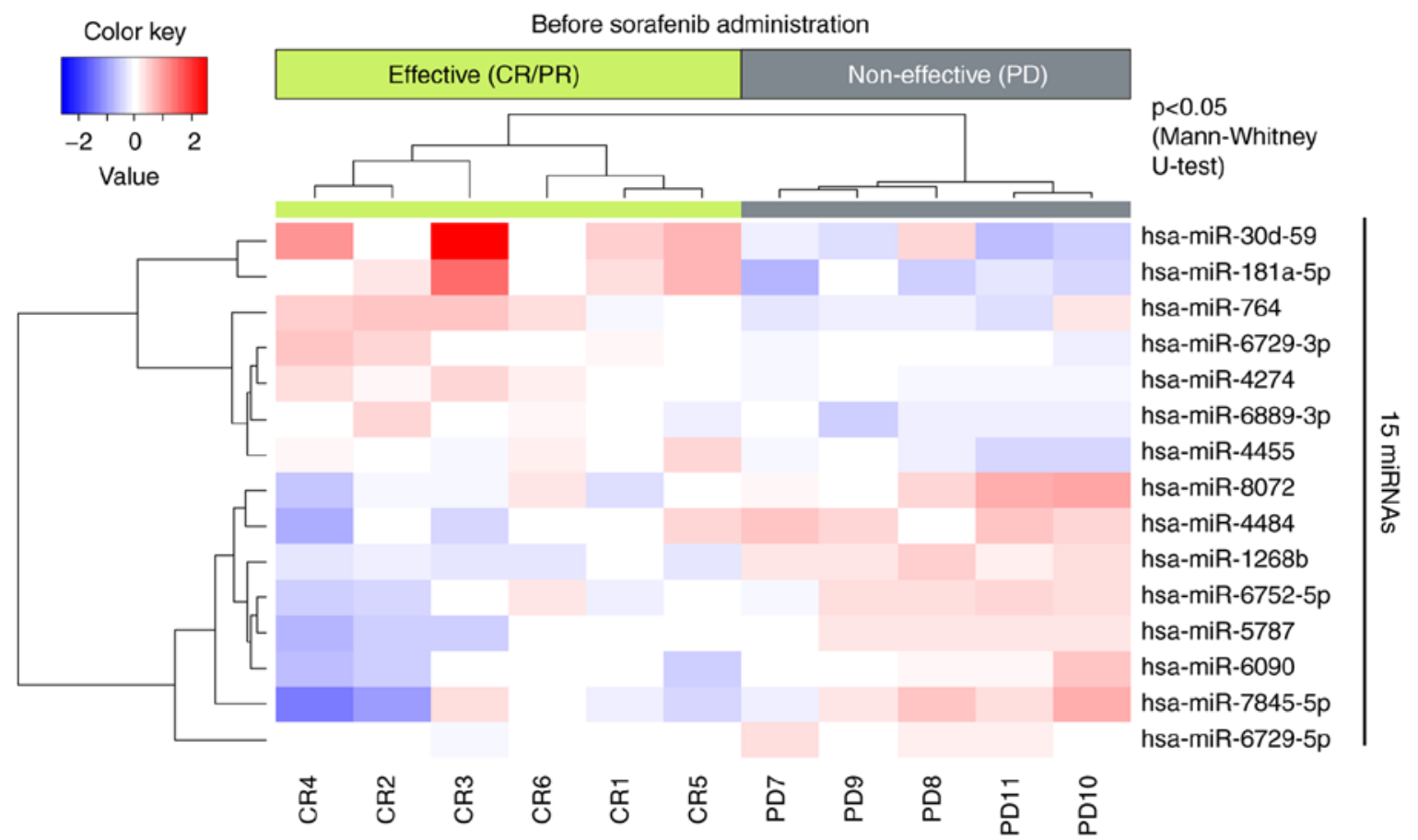

Figure 3. Cluster analysis of miRNA expression between the effective (CR/PR) and non-effective groups (PD) before sorafenib administration in vivo. The sorafenib-effective group formed a cluster separated from the non-effective group, 10 miRNAs were differentially expressed in sera of patients with HCC before sorafenib therapy between both groups. miRNA, microRNA; CR, complete response; PR, partial response; PD, progressive disease.

miR-224, and miR-10b-3p, were related to positive radiological responses in sorafenib-treated HCC patients (22). Additionally, hsa-miR-30d is associated with cell cycle arrest-related and apoptosis-induced molecules (23). Therefore, the down-regulation of hsa-miR-30d in HCC tissues might inhibit tumor growth through cell cycle arrest and apoptosis. On the other hand, secreted hsa-miR-30d might inhibit cell cycle arrest-related molecules in adjacent HCC tissues and induce non-tumor cell proliferation. These results, along with our data, suggest that miRNAs, including hsa-miR-30d, can serve as pivotal predictive biomarkers of the effectiveness of sorafenib therapy.

In addition, our study demonstrated that hsa-miR-30d was down-regulated in liver cancer cells, but some cell lines such as Li-7 and Hep3B, showed cell cycle inhibitory effects of sorafenib. The relationship between hsa-miR-30d down-regulation and direct inhibitory effects of sorafenib in liver cancer cell lines remains unclear. In our present study, although low hsa-miR-30d expression in the cells revealed inhibitory effects for Li-7 and Hep3B cells, active secretion of hsa-miR-30d from liver cancer cells was detected in the serum of HCC patients and medium of liver cancer cell lines. In order to confirm if hsa-miR-30d is involved in sorafenib therapy, we examined the hsa-miR-30d expression in the medium of liver cancer cell lines $48 \mathrm{~h}$ after sorafenib administration using real-time RT-PCR (Fig. S1). In Fig. S1, the hsa-miR-30d expression was significantly increased in the mediums of effective group as compared to those of non-effective group. This suggests our hypothesis that hsa-miR-30d does not induce direct inhibitory effect in the cell, but hsa-miR-30d secreted from liver cancer cells might be involved in the sensitivity of sorafenib for liver cancer cells.

The highlight of our study is that the expression levels of hsa-miR-30d diverged between cancer cell lines and serum 

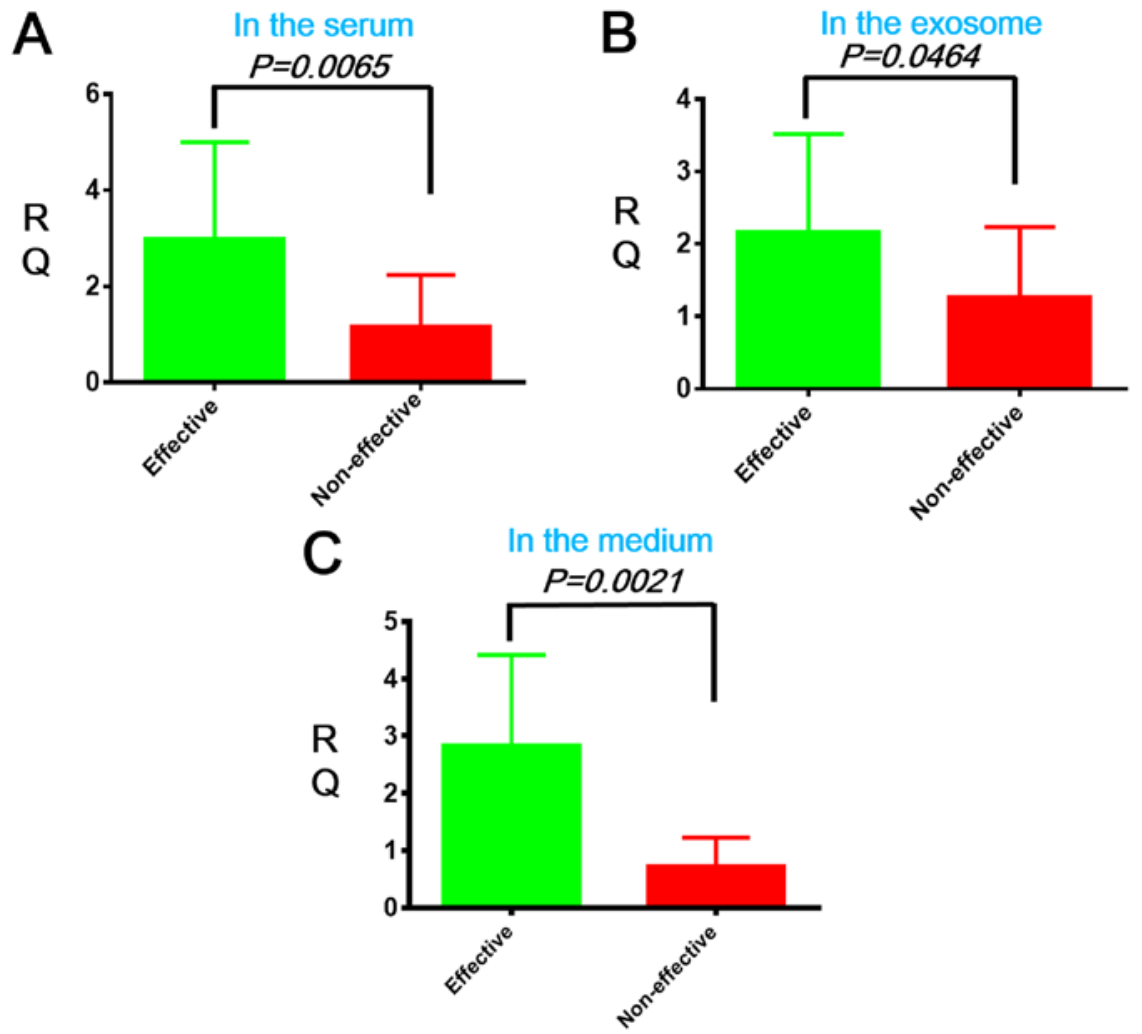

Figure 4. Quantification of hsa-miR-30d-5p in vitro and in vivo by reverse transcription-PCR. For the in vivo study, hsa-miR-30d-5p was upregulated in (A) the serum and (B) exosomes of the sorafenib-effective group when compared with the non-effective group before administration. (C) For the in vitro study, hsa-miR-30d-5p was upregulated in the medium of the sorafenib-effective group when compared with the non-effective group. miR, microRNA; RQ, relative quantification.

samples in HCC patients. Miquelestorena-Standley et al demonstrated that miR-152 was down-regulated in the serum of HCC patients when compared to that of non-HCC patients, while miR-152 expression in tissue did not change between HCC and non-HCC (24). In our study, hsa-miR-30d secretion was actively regulated by liver cancer cells independently from miRNA leakage due to liver damage, such as inflammation and tumor necrosis (Fig. 4; Table II). The results of Coenen-Stass et al (25) support our data by showing that some circulating miRNAs might be actively secreted and involved with the clinicopathological features of patients with HCC (26). It has also been demonstrated that miRNA leakage from hepatocytes, as in the case of miR-122, can easily be influenced by liver damage, including inflammation, steatosis, and fibrosis (27). Therefore, circulating miRNAs, such as miR-122, which are involved in hepatocyte damage, are not useful biomarkers to evaluate HCC treatment. hsa-miR-30d-5p could serve as a better and more specific biomarker of HCC because it is independent of inflammation and tumor necrosis.

The weakness of this study is the small number of HCC patients and lack of functional analysis of hsa-miR-30d. We could target several miRNAs for predictive markers for the sorafenib therapy, but the relationship between those miRNAs and clinical parameters, such as overall survival, progression free survival, and disease control rate, remains still unknown. In addition, direct inhibitory effect by hsa-miR-30d for liver cancer cells also remains elusive. Future study enrolling larger number of HCC patients and functional analysis of hsa-miR-30d will reveal more new evidences for sorafenib therapy.
In conclusion, hsa-miR-30d is up-regulated in the sera of HCC patients before sorafenib therapy and down-regulated in liver cancer cells. The secretion of hsa-miR-30d is actively controlled by HCC cells independent of miRNA leakage due to liver damage. Therefore, hsa-30d-5p might serve as a new predictive biomarker of sorafenib therapy outcomes in patients with HCC.

\section{Acknowledgements}

The authors would like to thank Ms. Kayo Hirose, Ms. Fuyuko Kokado and Ms. Keiko Fujikawa (Department of Gastroenterology and Neurology, Kagawa University Faculty of Medicine) for technical assistance during the microRNA array.

\section{Funding}

No funding was received.

\section{Availability of data and materials}

All data generated or analyzed during this study are included in this published article.

\section{Authors' contributions}

AM designed the concept of the present study. TK, AM, HI, KF, JT, KT, MN, KO, TT, TN, HY, KK, KO, YS, TH and TM performed outpatient service, obtained the informed 
consents, preserved the samples, and performed analysis and interpretation of data. AM, TK, KF, HI, AN and TM analyzed the serum microRNAs. TK and AM performed the in vitro experiments. TK and AM wrote the draft of the manuscript, and TM reviewed it. All authors read and approved the final manuscript.

\section{Ethics approval and consent to participate}

The present study was conducted in accordance with the ethical principles of the Declaration of Helsinki and approved by The Institutional Review Board of Kagawa University, Faculty of Medicine (Heisei-22-063). Informed consent to use the clinical data and samples for the present study was obtained from the patients or their relatives. For patients who died and had no relatives listed in their clinical records, opt-out methods were provided for the relatives of the dead participants by publishing a summary of this study on the university website.

\section{Patient consent for publication}

Not applicable.

\section{Competing interests}

The authors declare that they have no competing interests.

\section{References}

1. Morishita A and Masaki T: miRNA in hepatocellular carcinoma. Hepatol Res 45: 128-141, 2015.

2. Torre LA, Bray F, Siegel RL, Ferlay J, Lortet-Tieulent J and Jemal A: Global cancer statistics, 2012. CA Cancer J Clin 65: 87-108, 2015.

3. Lacaze L and Scotté M: Surgical treatment of intra hepatic recurrence of hepatocellular carcinoma. World J Hepatol 7: 1755-1760, 2015.

4. Imamura H, Matsuyama Y, Tanaka E, Ohkubo T, Hasegawa K, Miyagawa S, Sugawara Y, Minagawa M, Takayama T, Kawasaki S and Makuuchi M: Risk factors contributing to early and late phase intrahepatic recurrence of hepatocellular carcinoma after hepatectomy. J Hepatol 38: 200-207, 2003.

5. Venook AP, Papandreou C, Furuse J and de Guevara LL: The incidence and epidemiology of hepatocellular carcinoma: A global and regional perspective. Oncologist 15 (Suppl 4): S5-S13, 2010.

6. Kudo M: Systemic therapy for hepatocellular carcinoma: 2017 update. Oncology 93 (Suppl 1): S135-S146, 2017.

7. Llovet JM, Ricci S, Mazzaferro V, Hilgard P, Gane E, Blanc JF, de Oliveira AC, Santoro A, Raoul JL, Forner A, et al: Sorafenib in advanced hepatocellular carcinoma. N Engl J Med 359: 378-390, 2008

8. Pelosof L, Lemery S, Casak S, Jiang X, Rodriguez L, Pierre V, Bi Y, Liu J, Zirkelbach JF, Patel A, et al: Benefit-risk summary of regorafenib for the treatment of patients with advanced hepatocellular carcinoma that has progressed on Sorafenib. Oncologist 23: 496-500, 2018.

9. Belghiti J and Kianmanesh R: Surgical treatment of hepatocellular carcinoma. HPB (Oxford) 7: 42-49, 2005.

10. Masaki T: MicroRNA and hepatocellular carcinoma. Hepatol Res 39: 751-752, 2009.

11. Krek A, Grün D, Poy MN, Wolf R, Rosenberg L, Epstein EJ, MacMenamin P, da Piedade I, Gunsalus KC, Stoffel M and Rajewsky N: Combinatorial microRNA target predictions. Nat Genet 37: 495-500, 2005.
12. Meng F, Henson R, Wehbe-Janek H, Ghoshal K, Jacob ST and Patel T: MicroRNA-21 regulates expression of the PTEN tumor suppressor gene in human hepatocellular cancer. Gastroenterology 133: 647-658, 2007.

13. Gramantieri L, Ferracin M, Fornari F, Veronese A, Sabbioni S, Liu CG, Calin GA, Giovannini C, Ferrazzi E, Grazi GL, et al: Cyclin G1 is a target of miR-122a, a microRNA frequently down-regulated in human hepatocellular carcinoma. Cancer Res 67: 6092-6099, 2007.

14. Wong QW, Lung RW, Law PT, Lai PB, Chan KY, To KF and Wong N: MicroRNA-223 is commonly repressed in hepatocellular carcinoma and potentiates expression of Stathmin1. Gastroenterology 135: 257-269, 2008.

15. Varnholt H, Drebber U, Schulze F, Wedemeyer I, Schirmacher P, Dienes HP and Odenthal M: MicroRNA gene expression profile of hepatitis C virus-associated hepatocellular carcinoma. Hepatology 47: 1223-1232, 2008.

16. Vaira V, Roncalli M, Carnaghi C, Faversani A, Maggioni M, Augello C, Rimassa L, Pressiani T, Spagnuolo G, Di Tommaso L, et al: MicroRNA-425-3p predicts response to sorafenib therapy in patients with hepatocellular carcinoma. Liver Int 35: 1077-1086, 2015.

17. Gyöngyösi B, Végh É, Járay B, Székely E, Fassan M, Bodoky G, Schaff $Z$ and Kiss A: Pretreatment MicroRNA level and outcome in Sorafenib-treated hepatocellular carcinoma. J Histochem Cytochem 62: 547-555, 2014.

18. Livak KJ and Schmittgen TD: Analysis of relative gene expression data using real-time quantitative PCR and the 2(-Delta Delta C(T)) method. Methods 25: 402-408, 2001.

19. El-Serag HB: Hepatocellular carcinoma. N Engl J Med 365: 1118-1127, 2011

20. Kane RC, Farrell AT, Madabushi R, Booth B, Chattopadhyay S, Sridhara R, Justice R and Pazdur R: Sorafenib for the treatment of unresectable hepatocellular carcinoma. Oncologist 14: 95-100, 2009.

21. Nishida N, Arizumi T, Hagiwara S, Ida H, Sakurai T and Kudo M: MicroRNAs for the prediction of early response to sorafenib treatment in human hepatocellular carcinoma. Liver Cancer 6: 113-125, 2017.

22. Yoon EL, Yeon JE, Ko E, Lee HJ, Je JH, Yoo YJ, Kang SH, Suh SJ, Kim JH, Seo YS, et al: An explorative analysis for the role of serum miR-10b-3p levels in predicting response to sorafenib in patients with advanced hepatocellular carcinoma. J Korean Med Sci 32: 212-220, 2017.

23. Díez-Planelles C,Sánchez-LozanoP,Crespo MC,Gil-ZamoranoJ, Ribacoba R, González N, Suárez E, Martínez-Descals A, Martínez-Camblor P, Álvarez V, et al: Circulating microRNAs in Huntington's disease: Emerging mediators in metabolic impairment. Pharmacol Res 108: 102-110, 2016.

24. Miquelestorena-Standley E, Tallet A, Collin C, Piver E, De Muret A, Salamé E, Bourlier P, Kervarrec T, Guyétant S and Pagès JC: Interest of variations in microRNA-152 and -122 in a series of hepatocellular carcinomas related to hepatitis $\mathrm{C}$ virus infection. Hepatol Res 48: 566-573, 2018.

25. Coenen-Stass AM, Betts CA, Lee YF, Mäger I, Turunen MP, El Andaloussi S, Morgan JE, Wood MJ and Roberts TC: Selective release of muscle-specific, extracellular microRNAs during myogenic differentiation. Hum Mol Genet 25: 3960-3974, 2016.

26. Liu AM, Yao TJ, Wang W, Wong KF, Lee NP, Fan ST, Poon RT, Gao C and Luk JM: Circulating miR-15b and miR-130b in serum as potential markers for detecting hepatocellular carcinoma: A retrospective cohort study. BMJ Open 2: e000825, 2012.

27. Nakao K, Miyaaki $\mathrm{H}$ and Ichikawa T: Antitumor function of microRNA-122 against hepatocellular carcinoma. J Gastroenterol 49: 589-593, 2014.

This work is licensed under a Creative Commons Attribution-NonCommercial-NoDerivatives 4.0 International (CC BY-NC-ND 4.0) License. 